\title{
SLC26A4/PDS genotype-phenotype correlation in hearing loss with enlargement of the vestibular aqueduct (EVA): evidence that Pendred syndrome and non-syndromic EVA are distinct clinical and genetic entities
}

\author{
S P Pryor, A C Madeo, J C Reynolds, N J Sarlis, K S Arnos, W E Nance, Y Yang, C K Zalewski, \\ C C Brewer, J A Butman, A J Griffith
}

$\mathrm{E}$ nlargement of the vestibular aqueduct (EVA) and its contents, the endolymphatic sac and duct, is the most common radiologic malformation of the inner ear associated with sensorineural hearing loss. ${ }^{1}$ It may occur alone or in combination with an incomplete partition of the apical turn of the cochlea as part of a complex of malformations known as a Mondini deformity. ${ }^{2}$ Hearing loss in ears with EVA is typically pre- or perilingual in onset, sensorineural or mixed, and fluctuating or progressive. EVA may be unilateral or bilateral; asymmetry of the hearing loss and the anatomic defect is common in bilateral cases. ${ }^{3-5}$

EVA has been observed in Pendred syndrome (PS; MIM 274600), ${ }^{6}$ branchio-oto-renal syndrome (MIM 113650), ${ }^{7}$ CHARGE (MIM 214800), ${ }^{8}$ Waardenburg syndrome (MIM 193500, 193510, 600193, 606662), ${ }^{9}$ and distal renal tubular acidosis with deafness (MIM 267300). ${ }^{10}$ Familial non-syndromic hearing loss with EVA was described in $1996^{11}$ and numerous subsequent reports (DFNB4 (MIM 600791), enlarged vestibular aqueduct syndrome (MIM 603545)). EVA is always detected when the ears of individuals with PS are evaluated by both computed tomography (CT) and magnetic resonance imaging (MRI), and it has been estimated that PS may comprise up to $10 \%$ of prelingual deafness worldwide. ${ }^{3}{ }^{12} 13$ PS is inherited in an autosomal recessive manner and is comprised of bilateral hearing loss, EVA, and an iodine organification defect in the thyroid gland, which may lead to goitre. PS is clinically differentiated from non-syndromic EVA by the presence of the thyroid iodine organification defect because goitre is an incompletely penetrant feature of PS. ${ }^{3}$ When goitre does occur in PS, it is most often euthyroidal and not evident until the second decade of life. ${ }^{3121415}$ There can be intrafamilial variability of the goitre, and PS phenocopies with goitre due to other etiologies have been reported. ${ }^{16}$

The perchlorate discharge test can detect the thyroid iodine organification defect in PS, but has acquired a reputation as an unreliable diagnostic criterion due to its low specificity and significant variation in the criteria used for interpretation. ${ }^{1418} 18$ There has been no standardisation of iodine isotopes, doses, routes of administration, timing of perchlorate administration in relation to the iodine dose, or consensus on the definitive time point for measurement of discharge. Finally, the percent discharge criterion to differentiate normal from abnormal tests has varied widely among different studies.

Host factors can also confound the interpretation of the perchlorate discharge test. Discharge may be abnormal in patients with Hashimoto's thyroiditis, Graves' disease, cretinism, or intrathyroidal peroxidase deficiency. ${ }^{14}$ Previous surgical resection or radioablation of the thyroid, or recent

\section{Key points}

- Enlargement of the vestibular aqueduct (EVA) is a malformation of the inner ear associated with either non-syndromic or syndromic forms of sensorineural hearing loss, such as Pendred syndrome (PS). PS is differentiated from non-syndromic EVA by the presence of an iodine organification defect in the thyroid, which may lead to goitre. This defect can be detected with the perchlorate discharge test. SLC26A4 (PDS) mutations are detected in many cases of PS and nonsyndromic EVA, but no clear genotype-phenotype correlation has emerged from previous studies.

- We evaluated the clinical phenotype and SLC26A4 genotype of 39 patients with EVA from 31 families, and were able to definitively classify 29 subjects. All 11 PS subjects had two mutant SLC26A4 alleles, whereas all 18 non-syndromic EVA subjects had either one or zero SLC26A4 mutant alleles.

- PS is a genetically homogeneous disorder caused by biallelic SLC26A4 mutations.

- Haplotype analysis of sibships segregating non-syndromic EVA and one or zero SLC26A4 mutations indicates that at least some cases of non-syndromic EVA are associated with a single SLC26A4 mutation.

- The detection of a single mutant SLC26A4 allele is incompletely diagnostic without additional clinical evaluation to differentiate PS from non-syndromic EVA.

ingestion of thyrotropic medications, food, or liquids may also cause abnormal perchlorate discharge values, precluding a reliable test interpretation. ${ }^{18}$ Chronic dietary iodine intake may also theoretically affect discharge results. A complete medical history and serologic screening for Hashimoto's thyroiditis and Graves' disease, with thorough pre-test patient education, is required to identify these confounding factors and avoid a misleading discharge evaluation.

Abbreviations: ANOVA, one way analysis of variance; $C T$, computed tomography; EVA, enlargement of the vestibular aqueduct; FIESTA, fast induction employing steady state excitation; MRI, magnetic resonance imaging; PD, Parkinson disease; PS, Pendred syndrome; SNP, single nucleotide polymorphism; SSCP, single strand conformation polymorphism; STR, short tandem repeat; VA, vestibular aqueduct 
Recessive mutations of SLC26A4, which encodes the anion transporter pendrin, are the cause of PS. ${ }^{19}$ Over 90 mutations of SLC26A4 have been reported to date. These mutations are located throughout the coding region and include missense, nonsense, splice site, and frameshift mutations, as well as a large partial genomic deletion. ${ }^{13}$ The distributions of mutant SLC26A4 alleles differ significantly among various ethnic populations ${ }^{13}$; thus it is often necessary to sequence all 21 SLC26A4 exons in order to reliably identify mutant alleles.

EVA may also be associated with non-syndromic hearing loss, and familial cases have been consistent with recessive inheritance. ${ }^{11}$ The DFNB4 locus was originally mapped in a large consanguineous pedigree cosegregating SLC26A4 linked short tandem repeat (STR) markers and deafness that was originally believed to be non-syndromic. ${ }^{20}$ However, reascertainment of this kindred by the original authors reportedly revealed that the mutant phenotype included thyroid goitre and the affected individuals had PS. ${ }^{21}$ A large Indian family was subsequently reported to co-segregate non-syndromic EVA with homozygosity for a mutant SLC26A4 allele at the putative DFNB4 locus for non-syndromic recessive deafness. ${ }^{21}$ Although none of the affected individuals had a goitre, perchlorate discharge testing was not available to definitively rule out a thyroid iodine organification defect.

SLC26A4 mutations cannot be detected in about one third of patients with EVA, whereas only one mutant SLC26A4 allele is identified in another third. ${ }^{22-24}$ Because of the challenges in clinical evaluation of the PS thyroid phenotype, most studies have not reliably distinguished non-syndromic EVA from PS. Thus, a clear correlation of SLC26A4 genotype with thyroid phenotype has not emerged, suggesting that PS and non-syndromic EVA may be variant phenotypic manifestations of a single disorder. Alternatively, PS and nonsyndromic EVA may represent clinically and etiologically distinct entities. In order to distinguish between these hypotheses, we performed a rigorous phenotypic and SLC26A4 genotypic analysis of 39 EVA patients from 31 families. Our results provide compelling evidence that PS and non-syndromic EVA are clinically and etiologically distinct entities.

\section{METHODS \\ Subjects}

Our subjects consisted of 39 affected subjects with EVA and their unaffected relatives from 31 families. A total of $84 \%$ of these families were Caucasian. Affected individuals included 17 males and 22 females ranging in age from 2 to 59 years old. The eligibility criterion for an affected subject was EVA in at least one ear imaged by CT or MRI. EVA was defined as a vestibular aqueduct (VA) diameter exceeding $1.5 \mathrm{~mm}$ at the midpoint between the posterior cranial fossa and the vestibule of the inner ear, or a grossly malformed overall morphology of the VA. ${ }^{1}$ The study was approved by the joint NINDS/NIDCD Institutional Review Board (National Institutes of Health, Bethesda, MD) and written informed consent was obtained for all subjects.

\section{Clinical evaluation}

Each affected subject was evaluated at the NIH Clinical Center. This included a detailed medical history and physical examination (by SPP or AJG). Age appropriate audiometry was performed and past audiology records were reviewed when available. Urinalysis and renal ultrasound examinations were obtained in order to identify patients with renal abnormalities. Thyroid ultrasound was performed to determine presence or absence of goitre. Whenever possible, high resolution imaging of the inner ear was performed using a fast induction employing steady state excitation (FIESTA) and/or a T2 weighted, fast spin echo on a $1.5 \mathrm{~T}$ MRI system. Thyroid function tests, anti-thyroid antibody titres, a complete blood count, electrolyte levels, erythrocyte sedimentation rate, and a syphilis screen (RPR or FTA-ABS) were also obtained for each affected subject. Subjects with abnormal thyroid function or anatomy were evaluated by an endocrinologist to identify confounding PS phenocopies. Family history was obtained and genetic counselling provided (by ACM).

A perchlorate discharge test was performed on affected subjects with bilateral EVA who were able to maintain voluntary urinary continence. Due to the potential risks of radiation exposure with the perchlorate discharge test, we did not routinely test every subject with unilateral EVA since there are no reports of unilateral EVA associated with clinically confirmed PS. Subjects were required to discontinue any thyroid medications for the 6 weeks prior to testing; subjects resumed their medications if they developed symptoms of hypothyroidism, but this precluded them from undergoing the test. Subjects were kept nil per os on the day of the test. All tests were performed by the same technician and overseen by a nuclear medicine physician without knowledge of the patient's genotype. Iodine-123 was administered in capsule form and its uptake measured in the thyroid and thigh. Potassium perchlorate was orally administered $120 \mathrm{~min}$ after the iodine, and activity was measured in the thyroid and thigh at 150, 180, 240, and $300 \mathrm{~min}$ after iodine administration. Subtracted (thyroid minus thigh) measurements were corrected for decay and expressed as percent of peak iodine uptake.

Unaffected family members underwent history and physical examination, audiometry, and MRI of the inner ears. The latter evaluation was omitted for a few subjects who could not lie still or comfortably in the scanner.

\section{Molecular evaluation}

Venous blood or buccal swab samples were collected from subjects and DNA isolation was performed using the Gentra Puregene kit (Gentra Systems, Minneapolis, MN). The 21 SLC26A4 exons and their splice sites were PCR amplified from genomic DNA using Advantage polymerase (BD Biosciences Clontech, Palo Alto, CA) with conditions and primers as previously described. ${ }^{13}$ The coding exon of GJB2 was amplified and sequenced using the same conditions. GJB2 amplification primers were $5^{\prime}$-TGTGCATTCGTCTTTTCCA GAG-3' and 5'-TTGCCTCATCCCTCTCATGCTG-3'; additional primers $5^{\prime}$-CCAGGCTGCAAGAACGTGTG-3' and '5'-TGGGTT TTGATCTCCTCGAT-3' were used for sequencing. Amplification products were visually confirmed by agarose gel electrophoresis and treated with shrimp alkaline phosphatase and exonuclease I (USB, Cleveland, OH). Bidirectional sequencing reactions were performed with ABI BigDye Terminator kits, versions 1.0 or 3.0, and analysed on ABI 377 or 3730 sequencers (Applied Biosystems, Foster City, CA).

A DNA sample from each proband was used for nucleotide sequence analysis of all 21 exons of SLC26A4. When a mutation was identified in a proband, the corresponding exon was sequenced in each additional subject from that family. When a novel mutation created or removed a predicted restriction digestion site, the mutation was confirmed by a restriction endonuclease assay. A Caucasian diversity panel of 96 subjects (Coriell Cell Repositories, Camden, NJ) comprised the control genomic DNA samples.

\section{Haplotype analysis}

Families segregating EVA with zero or one mutant SLC26A4 allele were included in a haplotype analysis of $S L C 26 A 4$ linked STR genotypes as described..$^{13}$ STR markers were amplified by PCR with fluorescent dye labelled primers flanking 
microsatellite repeat markers D7S496, D7S2459, and D7S2456. The products were visualised using an ABI 377 sequencer, and genotype analysis was performed using Genescan and Genotyper software (Applied Biosystems, Foster City, CA). SLC26A4 intronic single nucleotide polymorphisms (SNPs) were directly sequenced as needed for haplotype confirmation.

\section{Zygosity analysis}

Samples from twinships in families 219 and 213 were analysed to determine zygosity. In each family, a panel of unlinked STR markers was amplified by PCR with fluorescent dye labelled primers flanking microsatellite repeats. The products were visualised using an ABI 377 or 3730 sequencer, and genotype analysis was performed using Genescan and Genotyper or Genemapper software (Applied Biosystems, Foster City, CA). In family 219, seven markers were analysed; in family 213, 14 markers were analysed.

\section{Statistical analysis}

Based on clinical criteria, we assigned each subject to one of three phenotypic groups: PS, non-syndromic EVA, or indeterminate. Subjects were assigned to the PS group if the EVA was bilateral and perchlorate discharge was abnormal (greater than 15\% discharge at $120 \mathrm{~min}$ after administration of perchlorate) or if there was a goitre with no other identifiable etiology and a perchlorate discharge test could not be performed. Subjects were classified as nonsyndromic if a perchlorate discharge test was normal or if EVA was unilateral. Subjects without perchlorate discharge data who could not be classified based on these criteria were assigned to the indeterminate phenotype group. Fisher's exact test was used to determine if there was a significant difference in the observed number of mutant SLC26A4 alleles in each subject in the PS and non-syndromic groups, as compared to that expected by chance. Fisher's exact test was also used to determine if there was a difference in EVA laterality (unilateral $v$ bilateral EVA) in the group with one or zero SLC26A4 mutations and the group with two mutations.

Patients with perchlorate discharge data available were assigned into one of three groups according to genotype: zero, one, and two mutant SLC26A4 alleles. One way analysis of variance (ANOVA) was used to determine if there was a difference in the percent of perchlorate discharge at each time point between the groups. Tukey's honestly significant difference (HSD) test was used for post hoc comparisons between groups.

\section{RESULTS}

We detected at least one SLC26A4 mutation in $28(72 \%)$ of 39 affected subjects from 20 (65\%) of 31 families (fig 1). Six of the 20 SLC26A4 mutations identified in our cohort are novel (table 1). One SNP, V402V, which does not result in amino acid substitution or predicted exonic splicing enhancer disruption, was identified in patient 1556. This SNP is not believed to be pathogenic. The most prevalent mutations in our cohort were L597S, E384G, and V138F, found in four, three, and three families, respectively. All of the participating parents were unaffected as determined by imaging and audiometric criteria, thus we observed no vertical transmission of EVA. In every study family for which one mutant SLC26A4 allele was identified and parental samples were available, we found that one of the parents was also heterozygous for the same mutant allele. Because SLC26A4 lies in a potentially imprinted region of chromosome 7 we examined the pedigrees segregating one mutant SLC26A4 allele but found no bias in the gender of the parent from whom the mutant allele was transmitted. We identified no de novo mutations in our cohort, although this possibility

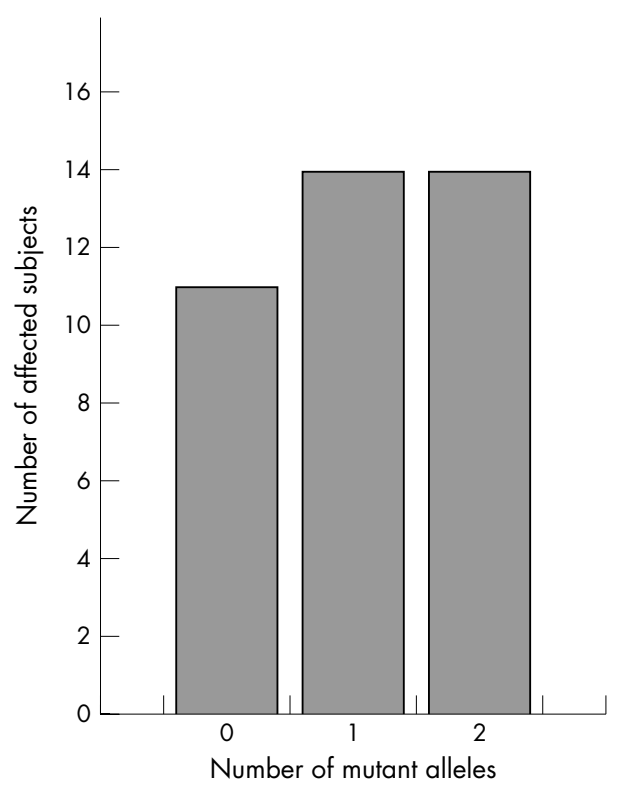

Figure 1 Number of mutant SLC26A4 alleles identified by bidirectional sequencing in each subject in entire EVA cohort. Two mutant alleles were identified in 14 affected individuals; 13 of these were compound heterozygotes and one was homozygous for the missense mutation V138F.

cannot be excluded in some of those families for which both parental samples were not available. The types or identities of SLC26A4 mutations did not appear to correlate with observed phenotypes.

We identified no pathogenic mutations and two coding polymorphisms in GJB2 among EVA subjects with zero or one SLC26A4 mutation. Unrelated subjects 1166 and 1426 were heterozygous for the M34T allele of GJB2, but neither subject's affected sibling carried M34T. Similarly, unrelated subjects 1426 and 1495 were heterozygous for the V153I allele of GJB2, although the affected sibling of 1426 was not a carrier. The discordant inheritance patterns indicate these polymorphisms do not contribute to the etiology of EVA.

Eleven subjects from nine families were classified as having PS, 18 subjects from 15 families as having nonsyndromic EVA, and 10 subjects from 10 families were indeterminate. Subjects classified as indeterminate were excluded from further analysis. Table 2 summarises the results of clinical and molecular evaluations in each affected subject. One subject (1618) had a goitre and a normal perchlorate discharge test, and an ultrasound evaluation revealed that the goitre consisted of a single large nodule in each lobe. Since these findings are inconsistent with the multinodular or smooth simple goitre of PS, subject 1618 was considered a phenocopy and classified as having nonsyndromic EVA without knowledge of her SLC26A4 genotype. All patients with PS had two mutant SLC26A4 alleles, while those with non-syndromic EVA had zero or one mutant SLC26A4 allele (fig 2). This difference was statistically significant $\left(\mathrm{p}=2.9 \times 10^{-8}\right)$.

One way ANOVA was performed on all perchlorate discharge values at each measured time point to determine if differences existed between patients with zero, one, or two mutant alleles. Significant differences were identified at each time point (30 min after perchlorate administration, $F=15.03, \mathrm{p}=0.0002 ; \mathrm{l} \mathrm{h}$ after perchlorate administration, $F=12.45, \mathrm{p}=0.0004 ; 2 \mathrm{~h}$ after perchlorate administration, $F=12.03, \mathrm{p}=0.0005 ; 3 \mathrm{~h}$ after perchlorate administration, $F=13.15, \mathrm{p}=0.0003$ ). Post hoc Tukey HSD tests on each time point revealed that this effect was due to significant 
Table 1 Novel SLC26A4 mutations detected in this study

\begin{tabular}{|c|c|c|c|c|c|}
\hline \multirow[b]{2}{*}{ Mutation } & \multirow[b]{2}{*}{ Nucleotide } & \multirow[b]{2}{*}{ Exon } & \multirow[b]{2}{*}{ Controls* } & \multicolumn{2}{|c|}{ Conservation of affected residue } \\
\hline & & & & Orthologst & Paralogsł \\
\hline IVS10-1 G>C & IVS10-1 G>C & IVS 10 & $0 / 168$ & - & - \\
\hline FS464/X487 & 1392delG & 12 & $0 / 176$ & - & - \\
\hline Q514R & $1541 A>G$ & 13 & $0 / 192$ & Yes & $1-6,9$ \\
\hline Y530S & $1589 \mathrm{~A}>\mathrm{C}$ & 14 & $0 / 192$ & Yes & $1,3-6,8,9$ \\
\hline V609G & $1826 \mathrm{~T}>\mathrm{G}$ & 17 & $0 / 144$ & No & No \\
\hline $\mathrm{R} 776 \mathrm{C}$ & $2326 C>T$ & 21 & $0 / 186$ & Yes & No \\
\hline
\end{tabular}

differences between the group with two mutant alleles and those with zero or one mutant alleles $(p<0.01)$. No significant difference existed in perchlorate discharge data at any time point between those with zero mutant alleles and those with one mutant allele $(\mathrm{p}>0.05)$.

Bilateral EVA was observed in association with zero, one, and two SLC26A4 mutations, and with both normal and abnormal perchlorate discharge results. In contrast, unilateral EVA was present only in subjects with zero or one SLC26A4 mutation, and was not observed in any subjects with an abnormal perchlorate discharge result (table 3 ). This difference was statistically significant $(\mathrm{p}=0.0049)$.

Haplotype analysis of SLC26A4 linked STR genotypes was performed in nine families segregating EVA with zero or one mutant SLC26A4 allele. Results were compatible with monogenic recessive inheritance of bi-allelic SLC26A4 mutations in five families, but were inconsistent with this inheritance pattern in four other families (133, 144, 213, and 219; fig 3). The apparent discordant inheritance in two of these four families (144 and 213) may be due to non-penetrance in unaffected siblings. Phenotypic discordance among the monozygotic twins of family 213 and SLC26A4 genotypic discordance among the affected dizygotic twins of family 219 indicate the influence of one or more non-genetic etiologic factors.

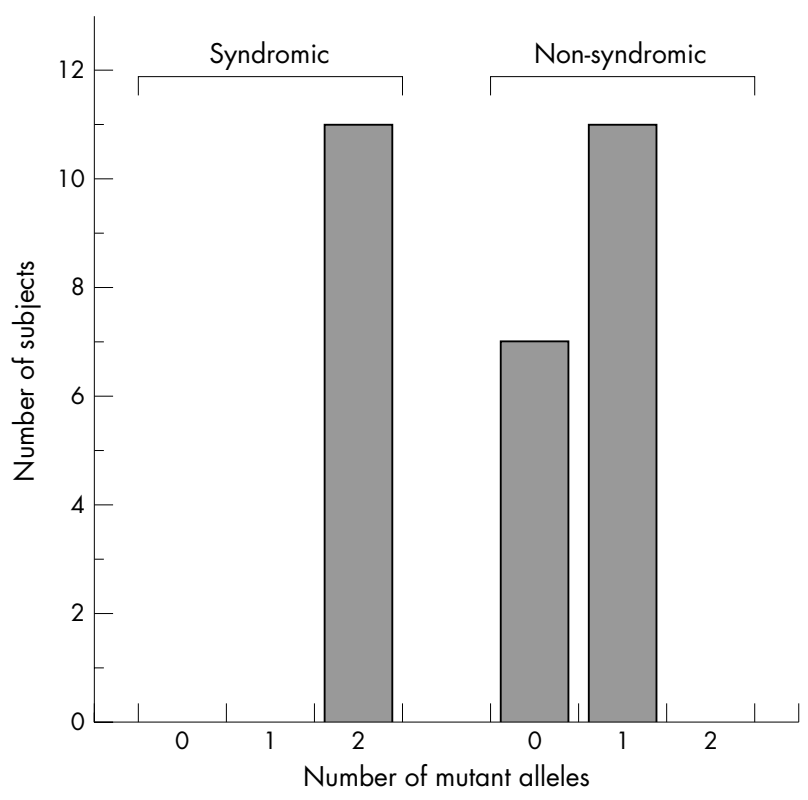

Figure 2 Genotype-phenotype correlation in subjects with EVA. All patients with PS had two mutant SLC26A4 alleles, while those with nonsyndromic EVA had zero or one mutant SLC26A4 allele. This difference was statistically significant $\left(p=2.9 \times 10^{-8}\right)$.

\section{DISCUSSION}

We have identified a significant correlation of phenotype with SLC26A4 genotype in a cohort of 39 patients with EVA and hearing loss. This correlation was not detected in previous studies, most likely because our uniform thyroid phenotypic analysis at a single facility permitted a more accurate classification. Inadequate periods of pre-test withdrawal of levothyroxine administration, or other undetected confounding factors such as ingestion of food or liquid or exogenous stable ("cold") iodine before or during the test, may have led to insufficient iodine uptake and unreliable discharge results in other studies. This may account for the normal perchlorate discharge results reported in other studies for some patients with EVA, goitre, and two SLC26A4 mutations. Finally, bidirectional nucleotide sequence analysis is more sensitive than single strand conformation polymorphism (SSCP) or denaturing high performance liquid chromatography for the detection of mutations. Increased sensitivity of our mutation detection method may therefore have facilitated the identification of the genotype-phenotype correlation.

Most of the SLC26A4 mutations identified in our cohort have been previously reported as pathogenic. We also detected six novel mutations that were not present in any normal control samples. The predicted effects of the novel splice site (IVS10-1G>C) and frameshift truncation (1392delG) mutations on the resulting mutant mRNA transcripts and proteins indicate they are likely to be pathogenic. Three of the four novel missense mutations, Q514R, Y530S, and R776C, affect residues that are conserved among SLC26A4 orthologs, indicating that these three mutations are also likely to be pathogenic. The residue affected by the sixth novel allele, V609G, is not evolutionarily conserved, raising the possibility that it may be a nondeleterious but rare polymorphism. Since V609G was identified in a single non-syndromic EVA subject, the strong statistical correlation of SLC26A4 genotype with phenotype is not diminished even if $\mathrm{V} 609 \mathrm{G}$ is not pathogenic.

Unfortunately, effective use of the perchlorate discharge test as a diagnostic tool has been limited due to its lack of specificity and the inconsistent criteria used for its interpretation. In the evaluation of our cohort, we used medical and family history, thyroid ultrasonography, serologic testing, and formal endocrinological evaluation to exclude potential sources of goitre or abnormal perchlorate results that might confound our analysis. The inconsistent criteria for the performance and interpretation of the perchlorate discharge test, in combination with their incomplete descriptions in the literature, have precluded the formulation of an appropriate criterion for the differentiation of normal from abnormal test results. Few studies have reported their precise perchlorate test methods and interpretation criteria; even fewer have published the quantitative results. Although we selected the time point of 240 min after iodine administration and $120 \mathrm{~min}$ after perchlorate administration based on 
Table 2 Phenotypes and genotypes of affected EVA subjects

\begin{tabular}{|c|c|c|c|c|c|c|c|c|}
\hline \multirow[b]{2}{*}{ Subject } & \multirow[b]{2}{*}{ Family } & \multirow[b]{2}{*}{ Sex } & \multirow[b]{2}{*}{ EVA* $^{*}$} & \multirow[b]{2}{*}{ Goitre } & \multirow[b]{2}{*}{ Perchlorate } & \multirow[b]{2}{*}{ Phenotype† } & \multicolumn{2}{|c|}{ SLC26A4 genotype } \\
\hline & & & & & & & Allele 1 & Allele 2 \\
\hline 1159 & 115 & $M$ & B & No & Not done & 1 & L236P & L445W \\
\hline 1163 & 115 & $\mathrm{~F}$ & B & No & Abnormal & PS & L236P & L445W $\ddagger$ \\
\hline 1171 & 115 & $\mathrm{~F}$ & B & Yes & Abnormal & PS & $\mathrm{L} 236 \mathrm{P}$ & $\mathrm{L} 445 \mathrm{~W}^{+}$ \\
\hline 1166 & 118 & $M$ & B & No & Normal & NS & L597S & wt \\
\hline 1167 & 118 & $M$ & $\mathrm{R}$ & No & Normal & NS & L597S & wt \\
\hline 1281 & 133 & $M$ & B & No & Normal & NS & R776C & wt \\
\hline 1282 & 133 & $\mathrm{~F}$ & $\mathrm{R}$ & No & Normal & NS & R776C & wt \\
\hline 1290 & 137 & $M$ & B & No & Not done & I & wt & wt \\
\hline 1303 & 142 & $M$ & B & No & Normal & NS & L445W & wt \\
\hline 1304 & 142 & $M$ & $\mathrm{R}$ & No & Normal & NS & L445W & wt \\
\hline 1343 & 144 & $M$ & B & No & Not done & I & wt & wt \\
\hline 1422 & 145 & $\mathrm{~F}$ & B & No & Not done & I & L597S & wt \\
\hline 1412 & 148 & $M$ & B & Yes & Abnormal & PS & $X 286$ & IVS8+1G >A \\
\hline 1387 & 151 & $\mathrm{~F}$ & B & No & Not done & I & G209V & Y530S \\
\hline 1377 & 153 & $\mathrm{~F}$ & B & Yes & Abnormal & PS & V138F & V138F \\
\hline 1425 & 156 & $M$ & B & No & Normal & NS & IVS8+1G >A & wt \\
\hline 1426 & 156 & $\mathrm{~F}$ & B & No & Not done & 1 & IVS8+1G>Ał & wt \\
\hline 1459 & 163 & $M$ & $\mathrm{~L}$ & No & Normal & NS & wt & wt \\
\hline 1445 & 166 & $\mathrm{~F}$ & $\mathrm{R}$ & No & Not done & NS & wt & wt \\
\hline 1447 & 167 & $\mathrm{~F}$ & B & No & Abnormal & PS & E384G & L597S \\
\hline 1460 & 171 & $\mathrm{~F}$ & B & No & Normal & NS & wt & wt \\
\hline 1464 & 175 & $\mathrm{~F}$ & B & No & Not done & 1 & wt & wt \\
\hline 1473 & 176 & $\mathrm{~F}$ & B & No & Normal & NS & L597S & wt \\
\hline 1470 & 177 & $M$ & B & No & Normal & NS & wt & wt \\
\hline 1495 & 182 & $\mathrm{~F}$ & B & No & Normal & NS & F335L & wt \\
\hline 1529 & 188 & $\mathrm{~F}$ & B & Yes & Not done & PS & L236P & 1536-1537delAG \\
\hline 1530 & 188 & $\mathrm{~F}$ & B & Yes & Not done & PS & $\mathrm{L} 236 \mathrm{P}$ & 1536-1537delAG $\ddagger$ \\
\hline 1556 & 193 & $\mathrm{~F}$ & B & Yes & Not done & PS & G209V & IVS10-1G >C \\
\hline 1604 & 194 & $M$ & B & No & Not done & i & V138F & 1343-1344insAGTC \\
\hline 1564 & 211 & $M$ & B & Yes & Abnormal & PS & FS464 & $\mathrm{Y} 530 \mathrm{H}$ \\
\hline 1583 & 213 & $\mathrm{~F}$ & $\mathrm{~L}$ & No & Not done & NS & wt & wt \\
\hline 1571 & 216 & $\mathrm{~F}$ & B & Yes & Not done & PS & V138F & E384G \\
\hline 1580 & 217 & $M$ & $\mathrm{R}$ & No & Normal & NS & E384G & wt \\
\hline 1590 & 218 & $M$ & B & No & Not done & 1 & V609G & wt \\
\hline 1598 & 219 & $\mathrm{~F}$ & B & Yes & Normal & NS & IVSI-2A $>G$ & wt \\
\hline 1599 & 219 & $\mathrm{~F}$ & B & No & Not done & 1 & wt & wt \\
\hline 1602 & 221 & $M$ & $\mathrm{~L}$ & No & Not done & NS & wt & wt \\
\hline 1618 & 225 & $\mathrm{~F}$ & L & Yes & Normal & NS & wt & wt \\
\hline 1616 & 227 & $\mathrm{~F}$ & B & Yes & Abnormal & PS & C565Y & Q514R \\
\hline
\end{tabular}

published methodologies, ${ }^{1825}$ any of our measured time points would have allowed discrimination between phenotypic groups. We chose a cutoff point of $15 \%$ discharge based on previously published reports. ${ }^{182627}$

We recognise that this classification scheme may not be accurate in all cases since perchlorate discharge values from patients with normal and abnormal iodine organification may overlap. If this were indeed the case, no single criterion would discriminate normal from abnormal values with 100\% sensitivity and specificity. We thus sought confirmation of the genotype-phenotype correlation without classifying individuals into syndromic or non-syndromic groups, asking instead whether there was a significant difference in thyroid iodine organification between the groups with zero, one, or two mutant SLC26A4 alleles. This approach revealed significant differences in the quantitatively measured rate of perchlorate discharge between the three groups at all measured time points, and confirmed that a genotypephenotype correlation does exist in EVA. Individuals with two mutant SLC26A4 alleles had a measurable iodine organification defect and PS, and those with zero or one mutant SLC26A4 alleles had indistinguishable perchlorate discharge values that fell within the range of normal, indicating the absence of an iodine organification defect. These individuals should be diagnosed as non-syndromic EVA.

The detection of one mutant SLC26A4 allele in over one half $(61 \%)$ of our non-syndromic EVA patients substantially exceeds the frequency in the general population as reported in the literature ${ }^{132223}$ and strongly suggests that monoallelic SLC26A4 mutations contribute to the pathogenesis of many cases of EVA. We observed no examples of vertical transmission of EVA in our cohort, and mutant SLC26A4 alleles never arose de novo in affected subjects. Whenever appropriate samples were available for testing, mutant SLC26A4 alleles in affected subjects were always found to be transmitted from one of the parents. Therefore a heterozygous SLC26A4 mutation by itself is not sufficient to cause EVA.

Overall, the prevalence and distribution of SLC26A4 mutations identified in our cohort was similar to that reported in previous studies with predominantly Caucasian subjects. Our strategy of bidirectional sequencing of PCR amplified exons and adjacent intronic sequences may have missed some deleterious mutations within SLC26A4 or its regulatory regions. Previous studies suggest that this strategy should identify approximately $90 \%$ of SLC26A4 mutations, as genomic rearrangement and deletion mutations are rare in SLC26A4. ${ }^{1322}$ Alternatively, individuals in whom zero or one mutant SLC26A4 allele was detected may have no additional SLC26A4 mutations, and some genetic or environmental cofactor might exist for non-syndromic EVA. We therefore conducted a haplotype analysis of markers linked to and within SLC26A4. In four of the nine families we examined, the results were inconsistent with simple monogenic autosomal recessive inheritance due to SLC26A4 mutations. This suggests that failure to detect pathogenic mutations in 


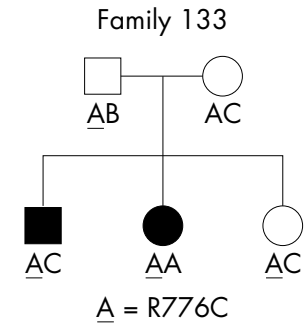

Family 219

Family 144

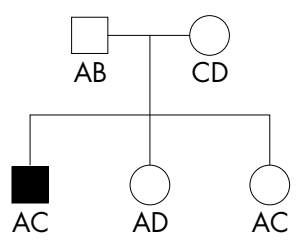

Family 213

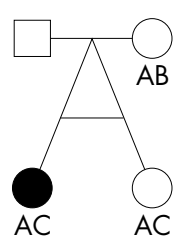

Figure 3 Inheritance of non-syndromic EVA and SLC26A4 alleles in four families. SLC26A4 linked STR haplotypes (denoted as A, B, C, or D) are underlined when linked to an SLC26A4 mutation. The affected sib pairs in families 133 and 219 demonstrate obligate recombinations of SLC26A4 alleles with EVA, based upon a model of simple monogenic inheritance of recessive SLC26A4 mutations. Single unaffected siblings in families 133, 144, and 213 may also be non-penetrant.

SLC26A4 cannot account for the observed genotype-phenotype correlation and strongly argues against a monogenic etiology for non-syndromic EVA due solely to recessive SLC26A4 mutations. These observations indicate that nonsyndromic EVA has a complex and possibly heterogeneous etiology. While SLC26A4 mutations are contributive in at least some cases, one or more environmental or genetic cofactors must also contribute to the development of non-syndromic EVA. This model is consistent with the variable auditory phenotypic expression of one SLC26A4 mutation among affected sib pairs in families 118 and 142 .

The FREAC6/FOXI1 gene has been proposed as a possible etiologic factor for PS. ${ }^{28}$ In our cohort, we found no evidence for etiologic heterogeneity in PS: two mutant SLC26A4 alleles were identified in each patient with PS. Preliminary FREAC6 sequencing results in our cohort suggest that mutations in this gene are not a major contributing factor to the development of non-syndromic EVA either (data not shown). Other candidate cofactors for non-syndromic EVA are genes underlying syndromes known to include EVA such as branchio-oto-renal syndrome, ${ }^{7}$ CHARGE, ${ }^{8}$ Waardenburg syndrome, ${ }^{9}$ and distal renal tubular acidosis with deafness. ${ }^{10}$ Our affected subjects did not have phenotypic manifestations of these syndromes, in spite of thorough physical, laboratory, and radiologic examinations specifically to detect such features. Non-syndromic EVA may nevertheless result from mono-, di- or multigenic inheritance of mutations in these or other genes alone or in combination with heterozygous SLC26A4 mutations. Analogous etiologic mechanisms appear to underlie other disorders such as Parkinson disease (PD) and Crohn's disease. ${ }^{29}{ }^{30}$ For example, a rare, juvenile onset form of PD can be inherited as an autosomal recessive trait caused by biallelic mutations in the parkin gene. In contrast, a more prevalent, later onset PD phenotype is often associated with a single mutant allele of parkin as part of a complex trait. ${ }^{29}$

Our study has confirmed that PS is a genetically homogeneous, autosomal recessive disorder caused by mutations in SLC26A4. We have demonstrated that PS and

Table 3 Summary of phenotypes and genotypes of EVA subjects

\begin{tabular}{lllll}
\hline & \multirow{2}{*}{$\begin{array}{l}\text { Perchlorate } \\
\text { discharge } \\
\text { result }\end{array}$} & \multicolumn{3}{l}{$\begin{array}{l}\text { Number of mutant SLC26A4 } \\
\text { alleles per subject }\end{array}$} \\
\cline { 3 - 5 } \cline { 3 - 4 } & Zero & One & Two \\
\hline Unilateral & Normal & 2 & 3 & 0 \\
Unilateral & Abnormal & 0 & 0 & 0 \\
Unilateral & Unknown & 3 & 1 & 0 \\
Bilateral & Normal & 2 & 7 & 0 \\
Bilateral & Abnormal & 0 & 0 & 7 \\
Bilateral & Unknown & $4^{*}$ & $3^{*}$ & $7 \dagger$ \\
\hline
\end{tabular}

*One subject had a sibling with a normal perchlorate discharge result; tfive subjects would likely have had an abnormal perchlorate discharge result: four had a goitre, and a fifth had two siblings with abnormal perchlorate discharge results.

non-syndromic EVA are clinically and genetically distinct entities, and the possibility that non-syndromic recessive deafness DFNB4 may be a non-existent phenotype. These conclusions have significant implications for the interpretation of clinical testing for SLC26A4 mutations. First, one mutant SLC26A4 allele cannot be considered diagnostic for PS. Second, all 21 exons should be evaluated in order to make the critical diagnostic distinction between one (or zero) and two mutant alleles. Third, the detection of two SLC26A4 mutant alleles may obviate the need for perchlorate discharge testing to diagnose PS, but SLC26A4 testing cannot supplant this clinical evaluation to definitively differentiate nonsyndromic EVA from PS in patients with one (or zero) detectable SLC26A4 mutations. The identification of etiologic cofactors for non-syndromic EVA would provide insight into its pathogenesis and the design of clinical or genetic evaluations for its diagnosis and provide for more accurate recurrence risk assessment.

\section{ACKNOWLEDGEMENTS}

We thank the study families for their participation, the NIDCD clinic staff, Mary Ann Mastroianni, Larry Shotland, Yvonne SzymkoBennett, Stephanie Moran, Angela Stuber, Nick Patronas, Monica Skarulis, Clara Chen, and Tom Shawker for assistance in clinical evaluations, Jim Thomsen, David Eisenman, Arti Pandya, Jeff Kim, Alex Arts, John Niparko and others for referral of study subjects, Robert Morell for zygosity analysis of family 213, Aqeel Chowdhry and Kristin Lubas for technical assistance, Hong-Joon Park and Lorraine Everett for technical advice, and Tom Friedman, Rob Morell, and Les Biesecker for critical review of the manuscript.

\section{Authors' affiliations}

S P Pryor, A C Madeo, C K Zalewski, C C Brewer, A J Griffith, Hearing Section, National Institute on Deafness and Other Communication Disorders, National Institutes of Health, Bethesda, MD, USA

S P Pryor, Office of the Clinical Director, National Institute on Deafness and Other Communication Disorders, National Institutes of Health, Bethesda, MD, USA

J C Reynolds, Nuclear Medicine Department, Warren G. Magnuson Clinical Center, National Institutes of Health, Bethesda, MD, USA

N J Sarlis*, Clinical Endocrinology Branch, National Institute of Diabetes and Digestive and Kidney Diseases, National Institutes of Health, Bethesda, MD, USA

K S Arnos, Department of Biology, Gallaudet University, Washington, DC, USA

W E Nance, Department of Human Genetics, Medical College of Virginia, Virginia Commonwealth University, Richmond, VA, USA Y Yang, A J Griffith, Section on Gene Structure and Function, Laboratory of Molecular Genetics, National Institute on Deafness and Other Communication Disorders, National Institutes of Health, Rockville, MD, USA

J A Butman, Diagnostic Radiology Department, Warren G. Magnuson Clinical Center, National Institutes of Health, Bethesda, MD, USA 
This study was supported by NIDCD/NIH intramural research funds 1-Z01-DC-000060 and 1-Z01-DC-000064.

Conflict of interest: none declared.

${ }^{*}$ Current address: Department of Endocrine Neoplasia and Hormonal Disorders, The University of Texas - M. D. Anderson Cancer Center, Houston, TX, USA.

Correspondence to: $\operatorname{Dr}$ A J Griffith, NIDCD/NIH, 5 Research Court, Room 2A-01, Rockville, MD 20850, USA; griffita@nidcd.nih.gov

Received 17 June 2004

Revised version received 11 August 2004

\section{REFERENCES}

1 Valvassori GE, Clemis JD. The large vestibular aqueduct syndrome Laryngoscope 1978;88(5):723-8.

2 Mondini C. Anatomica surdi nati sectio. In: De Bononiensi scientarium et artium instituto atque academia commentarii. Bonaniae, 1791:VIl:419-28.

3 Fraser GR. Association of congenital deafness with goitre (Pendred's syndrome): a study of 207 families. Ann Hum Genet 1965;28:201-49.

4 Jackler RK, De La Cruz A. The large vestibular aqueduct syndrome. Laryngoscope 1989:99(12):1238-43.

5 Madden C, Halsted M, Benton C, Greinwald J, Choo D. Enlarged vestibular aqueduct syndrome in the pediatric population. Otol Neurotol 2003;24(4):625-32.

6 Phelps PD, Coffey RA, Trembath RC, Luxon LM, Grossman AB, Britton KE, Kendall-Taylor P, Graham JM, Cadge BC, Stephens SG, Pembrey ME, Reardon W. Radiological malformations of the ear in Pendred syndrome. Clin Radiol 1998;53(4):268-73

7 Chen A, Francis M, Ni L, Cremers CW, Kimberling WJ, Sato Y, Phelps PD, Bellman SC, Wagner MJ, Pembrey M, Smith RJH. Phenotypic manifestations of branchio-oto-renal syndrome. Am J Med Genet 1995;58(4):365-70.

8 Bauer PW, Wippold FJ 2nd, Goldin J, Lusk RP. Cochlear implantation in children with CHARGE association. Arch Otolaryngol Head Neck Surg 2002; 128(9): 1013-7

9 Madden C, Halsted MJ, Hopkin RJ, Choo DI, Benton C, Greinwald JH Jr. Temporal bone abnormalities associated with hearing loss in Waardenburg syndrome. Laryngoscope 2003;113(11):2035-41.

10 Berrettini S, Neri E, Forli F, Panconi M, Massimetti M, Ravecca F, SellariFranceschini S, Bartolozzi C. Large vestibular aqueduct in distal renal tubula acidosis. High-resolution MR in three cases. Acta Radiol 2001;42(3):320-2.

11 Griffith AJ, Arts A, Downs C, Innis JW, Shepard NT, Sheldon S, Gebarski SS Familial large vestibular aqueduct syndrome. Laryngoscope 1996;106(8):960-5

12 Reardon W, Coffey R, Phelps PD, Luxon LM, Stephens D, Kendall-Taylor P, Britton KE, Grossman A, Trembath R. Pendred syndrome-100 years of underascertainment? QJM 1997:90(7):443-7.

13 Park HJ, Shaukat S, Liu XZ, Hahn SH, Naz S, Ghosh M, Kim HN, Moon SK Abe S, Tukamoto K, Riazuddin S, Kabra M, Erdenetungalag $R$ Radnaabazar J, Khan S, Pandya A, Usami SI, Nance WE, Wilcox ER, Griffith AJ. Origins and frequencies of SLC26A4 (PDS) mutations in east and south Asians: global implications for the epidemiology of deafness. J Med Genet 2003;40(4):242-8.
14 Morgans ME, Trotter WR. Association of congenital deafness with goitre: the nature of the thyroid defect. Lancet 1958;1:607-9. 15 Johnsen T, Larsen C, Friis J, Hougaard-Jensen F. Pendred's syndrome.
Acoustic, vestibular and radiological findings in 17 unrelated patients. $J$ Laryngol Otol 1987; 101(11):1187-92.

16 Kopp P, Arseven OK, Sabacan L, Kotlar T, Dupuis J, Cavaliere H, Santos CL, Jameson JL, Medeiros-Neto G. Phenocopies for deafness and goiter development in a large inbred Brazilian kindred with Pendred's syndrome associated with a novel mutation in the PDS gene. J Clin Endocrinol Metab 1999;84(1):336-41

17 Niepomniszcze H, Coleoni AH, Degrossi OJ, Scavini LM, Curutchet HP. Biochemical studies on the iodine organification defect of Pendred's syndrome. Acta Endocrinol (Copenh) 1978;89(1):70-9.

18 Wolff J. Perchlorate and the thyroid gland. Pharmacol Rev 1998;50(1):89-105

19 Everett LA, Glaser B, Beck JC, Idol JR, Buchs A, Heyman M, Adawi F, Hazani E, Nassir E, Baxevanis AD, Sheffield VC, Green ED. Pendred syndrome is caused by mutations in a putative sulphate transporter gene (PDS). Nat Genet 1997;17(4):411-22.

20 Baldwin CT, Weiss S, Farrer LA, De Stefano AL, Adair R, Franklyn B, Kidd KK, Korostishevsky M, Bonne-Tamir B. Linkage of congenital, recessive deafness (DFNB4) to chromosome 7q31 and evidence for genetic heterogeneity in the Middle Eastern Druze population. Hum Mol Genet 1995;4(9): 1637-42.

21 Li XC, Evereft LA, Lalwani AK, Desmukh D, Friedman TB, Green ED, Wilcox ER. A mutation in PDS causes non-syndromic recessive deafness. Nat Genet $1998 ; 18(3): 215-7$

22 Campbell C, Cucci RA, Prasad S, Green GE, Edeal JB, Galer CE, Karniski LP, Sheffield VC, Smith RJ. Pendred syndrome, DFNB4, and PDS/SLC26A4 identification of eight novel mutations and possible genotype-phenotype correlations. Hum Mutat 2001;17(5):403-11.

23 Coyle B, Reardon W, Herbrick JA, Tsui LC, Gausden E, Lee J, Coffey R, Grueters A, Grossman A, Phelps PD, Luxon L, Kendall-Taylor P, Scherer SW, Trembath RC. Molecular analysis of the PDS gene in Pendred syndrome. Hum Mol Genet 1998;7(7):1 105-12.

24 Gonzalez Trevino O, Karamanoglu Arseven O, Ceballos CJ, Vives VI, Ramirez RC, Gomez VV, Medeiros-Neto G, Kopp P. Clinical and molecular analysis of three Mexican families with Pendred's syndrome. Eur J Endocrinol $2001 ; 144(6): 585-93$.

25 Stewart RD, Murray IP. An evaluation of the perchlorate discharge test. J Clin Endocrinol Metab 1966;26(10):1050-8.

26 Fraser GR, Morgans ME, Trotter WR. The syndrome of sporadic goitre and congenital deafness. Q J Med 1960;29:279-95.

27 Trotter WR. Diseases of the thyroid. Oxford: Blackwell Scientific, 1962

28 Hulander M, Kiernan AE, Blomqvist SR, Carlsson P, Samuelsson EJ, Johansson BR, Steel KP, Enerback S. Lack of pendrin expression leads to deafness and expansion of the endolymphatic compartment in inner ears of Foxi1 null mutant mice. Development 2003;130(9):2013-25.

29 Pankratz N, Nichols WC, Uniacke SK, Halter C, Murrell J, Rudolph A, Shults CW, Conneally PM, Foroud T. Genome-wide linkage analysis and evidence of gene-by-gene interactions in a sample of 362 multiplex Parkinson disease families. Hum Mol Genet 2003;12(20):2599-608.

30 van Heel DA, Dechairo BM, Dawson G, McGovern DP, Negoro K, Carey AH, Cardon LR, Mackay I, Jewell DP, Lench NJ. The IBD6 Crohn's disease locus demonstrates complex interactions with CARD 15 and IBD5 disease-associated variants. Hum Mol Genet 2003;12(20):2569-75. 